Proceedings

\title{
Inclusion of Montelukast in y-Cyclodextrin: Presenting a Mechanochemical Route to Improve Drug Stability and Solubility ${ }^{\dagger}$
}

\author{
Jéssica S. Barbosa ${ }^{1,2}$, Karyna Lysenko ${ }^{1}$, Filipe A. Almeida Paz ${ }^{2}$ and Susana Santos Braga 1,* \\ 1 Department of Chemistry, LAQV-REQUIMTE, University of Aveiro, 3810-193 Aveiro, Portugal; \\ jessicambarbosa@ua.pt (J.S.B.); karynalysenko@ua.pt (K.L.) \\ 2 Department of Chemistry, CICECO-Aveiro Institute of Materials, University of Aveiro, \\ 3810-193 Aveiro, Portugal; filipe.paz@ua.pt \\ * Correspondence: sbraga@ua.pt \\ + Presented at the 1st International Electronic Conference on Pharmaceutics, 1-15 December 2020; \\ Available online: https://iecp2020.sciforum.net/.
}

Citation: Barbosa, J.S.; Lysenko, K.; Almeida Paz, F.A.; Braga, S.S. Inclusion of Montelukast in y-Cyclodextrin: Presenting a Mechanochemical Route to Improve Drug Stability and Solubility. Proceedings 2021, 78, 18. https://doi.org/10.3390/ IECP2020-08717

Published: 1 December 2020

Publisher's Note: MDPI stays neutral with regard to jurisdictional claims in published maps and institutional affiliations.

Copyright: (c) 2020 by the authors. Licensee MDPI, Basel, Switzerland. This article is an open access article distributed under the terms and conditions of the Creative Commons Attribution (CC BY) license (http://creativecommons.org/licenses /by/4.0/).

\begin{abstract}
Montelukast sodium (MLK) is a worldwide antiasthmatic drug. Commercial formulations still have some issues with solubility and instability to light and humidity. To overcome them, the present work reports inclusion compounds of MLK and $\gamma$-cyclodextrin $(\gamma$-CD). As a molecular capsule, CDs have the ability to protect the inclusion guest from degradation, enhance its solubility and alter the pharmacokinetic parameters. MLK $\gamma-C D$ inclusion compounds were prepared by mechanochemistry. Without using any solvent, $\gamma$-CD was pre-milled and then co-milled with an equimolar quantity of MLK in a ball mill at 600 cycles. $\mathrm{min}^{-1}$. After $120 \mathrm{~min}$ of milling, the formation of MLK· $\gamma$-CD inclusion compounds was confirmed by powder $\mathrm{X}$-ray diffraction and scanning electron microscopy. Additional studies, performed under pharmacopeia guidelines, showed that the prepared MLK· $\gamma-\mathrm{CD}$ inclusion compounds can indeed increase the dissolution of MLK when in ultrapure water or simulated intestinal fluid (without pancreatin). This way, the MLK· $\gamma-\mathrm{CD}$ inclusion compounds that are presented in this work are a promising solution for improving the therapeutic effectiveness of MLK.
\end{abstract}

Keywords: montelukast; cyclodextrins; mechanochemistry; inclusion compounds; drug solubility

\section{Introduction}

Montelukast sodium (MLK) is a widely used antiasthmatic agent [1-5] that can also be prescribed for other conditions, such as the treatment of allergic bronchopulmonary aspergillosis or chronic obstructive pulmonary disease (COPD) [6,7]. By blocking the cysteinyl-leukotriene 1 receptors, this active pharmaceutical ingredient (API) is able to significantly improve the lung function of these patients $[1,8]$.

Nowadays, there are already three different dosage formulations of MLK on the market: tablets, chewable tablets and granules. Given this offer, the most suitable formulation can be chosen according to the patient's needs concerning drug administration. For instance, MLK granule formulation is quite useful for 
administering to small babies and elderly patients, which could otherwise be a difficult challenge $[9,10]$. Nonetheless, and despite their proven efficiency, MLK formulations still present some limitations to their use, namely the poor solubility of the molecule in water $(100-1000 \mathrm{mg} / \mathrm{mL})$, as well as its instability to light, temperature, humidity and oxidation [9,11-13]. In an attempt to overcome these limitations, numerous approaches have been used, among them the use of cyclodextrins (CDs) as molecular carriers $[9,12,14,15]$. Cyclodextrins are a class of water-soluble cyclic oligosaccharides, well-known for the capacity to accommodate in their cavity a diversified class of guest molecules to form inclusion compounds (ICs). In the pharmaceutical industry, cyclodextrin inclusion com pounds are of particular interests because they can protect the included APIs from degradation and they can modify their physicochemical properties and pharmacokinetic parameters [16-19].

In this work, we report the inclusion compounds of MLK and gammacyclodextrin $(\gamma-C D)$, which has been proven to have the ideal cavity size for the inclusion of this guest. Besides, and in contrast with the common procedures $[12,20,21], \gamma$-CD.MLK is herein prepared by mechanochemical grinding, without using any solvent. This method applied to MLK was first described by us in a previous study [22] and it is gaining growing interest in pharmaceutical and organic chemistry applications [23]. In the end, we expect that the preparation of $\gamma$-CD.MLK will modulate the physicochemical properties of MLK, in particular its solubility and stability. Upon preliminary results described in [22], in this study pharmacopeia guidelines will be used to determine the physicochemical properties of $\gamma$-CD.MLK when compared with the pure drug.

\section{Experimental Section}

\subsection{Materials}

$\gamma$-CD heptahydrate $(\mathrm{MW}=1423.11$ ), produced by Wacker Chemie (in Eddyville, Iowa, USA) with the commercial name Cavamax W8, was kindly donated by Ashland Industries Deutschland Gmbh (Düsseldorf, Germany). Montelukast sodium ( $>98 \%$ of purity, MW $=608.17$ ), hereafter denominated MLK, was obtained from TCI or gently provided by Ashland (being produced by Ria International, Mumbai, India).

\subsection{Equipment}

Ball milling was carried out in a Philips MiniMill planetary apparatus, working at a velocity of 600 cycles $\mathrm{min}^{-1}$. Samples were loaded into $50 \mathrm{~mL}$ calcium-doped zirconia grinding jars, each containing two yttrium-doped zirconia milling balls with $1 \mathrm{~cm}$ of diameter.

Laboratory Powder X-ray Diffraction (PXRD) data were collected at ambient temperature on an Empyrean PANalytical diffractometer, with working wavelengths of $\lambda 1=1.540598 \AA$ and $\lambda 2=1.544426 \AA$ ( $\mathrm{Cu} \mathrm{K} \alpha 1,2 \mathrm{X}$ radiation), equipped with an PIXcel 1D detector and a flat-plate sample holder in a BraggBrentano para-focusing optics configuration $(45 \mathrm{kV}, 40 \mathrm{~mA})$. Intensity data were collected by the step-counting method (step $0.01^{\circ}$ ), in continuous mode, in the ca. $3.5 \leq 2 \theta^{\circ} \leq 50^{\circ}$ range.

Scanning Electron Microscopy (SEM) images were acquired with a Hitachi SU-70 Schottky emission instrument, working at $10 \mathrm{kV}$. Samples were prepared by deposition on aluminum sample holders followed by carbon coating in an 
Emitech K950X carbon evaporator. Energy-dispersive X-ray spectroscopy (EDS) mapping images were recorded using a Bruker QUANTAX 400 microanalysis system.

UV-Vis spectroscopy measurements for the studies of montelukast aqueous dissolution were conducted on a UV-2501 PC Shimadzu spectrometer, at a working wavelength of $346 \mathrm{~nm}$.

\subsection{Preparation of $\gamma$-CD $M L K$ by Mechanochemistry}

$\gamma$-CD.MLK was prepared from a mixture containing amorphous $\gamma$-CD and MLK as received from the manufacturer, under the experimental conditions previously described by Barbosa et al. [22].

\subsection{Dissolution of $\gamma$-CD.MLK versus Pure MLK: Preliminary In Vitro Studies}

The assays on the dissolution rate of $\gamma$-CD.MLK and pure MLK, when in ultrapure water, were performed as previously described by Barbosa et al. [22].

\subsection{Dissolution of $\gamma$-CD.MLK versus Pure MLK: According to Pharmacopeia Guidelines}

The dissolution profile of $\gamma$-CD·MLK and pure MLK were analysed under (1) ultrapure water, (2) ultrapure water with $0.5 \%(\mathrm{~m} / \mathrm{v})$ of sodium dodecyl sulphate (SDS), (3) simulated intestinal fluid (without pancreatin), with $\mathrm{pH}$ buffered at 6.8, and (4) acetate buffer, $\mathrm{pH} 4.5$; and according to Pharmacopeia guidelines. For this, a glass vessel with one liter capacity was filled with $900 \mathrm{~mL}$ of one of the aforementioned media. The vessel was immersed in a water bath of suitable dimensions, which allows for maintaining not only a temperature of $37 \pm 0.5^{\circ}$, but also a constant and smooth flow of water during the tests. In addition to this, the medium inside the container was kept under continuous stirring with a rotor and a shaft with an agitation paddle attached to it.

$10 \mathrm{mg}$ of pure MLK, or an amount of $\gamma$-CD.MLK IC that corresponds to the same value of the pure drug, were added to the medium inside the container, which was then covered to delay medium evaporation. Aliquots were then collected at 5, 10, 20 and $30 \mathrm{~min}$, for absorbance measurements under UV-Vis spectroscopy.

\section{Results and Discussion}

The preparation of $\gamma$-CD.MLK by co-milling reproduced previously described conditions [22]. In this work, we complement the previous work with details on the impact of inclusion on the physicochemical properties of MLK.

\subsection{Preparation of IC through Mechanochemistry}

Mechanochemical preparation of $\gamma$-CD.MLK followed the procedures described in Barbosa et al. [22]. The efficacy of this method is evaluated, on a first approach, by powder diffraction. As seen in Figure 1, upon 120 min of mechanical grinding an amorphous compound, with no reflections on its diffractogram, was obtained. This provides initial evidence of the formation of $\gamma$-CD.MLK because it is a typical feature for ICs prepared by mechanochemistry [24-26].

In addition, SEM images showed that in the physical mixture of MLK and amorphous $\gamma$-CD there are two distinct morphologies (Figure 2a). While MLK appears as an agglomerate of spheres, $\gamma$-CD particles present irregular shapes and sizes, with round edges. EDS mapping of this mixture further confirms the 
presence of the characteristic elements of MLK, namely $\mathrm{S}, \mathrm{Na}$ and $\mathrm{Cl}$, only in the agglomerates (Figure 2b). Upon 120 min of grinding, a uniform distribution of MLK was reached throughout the sample, confirming complete interaction between the two components (Figure 2d).
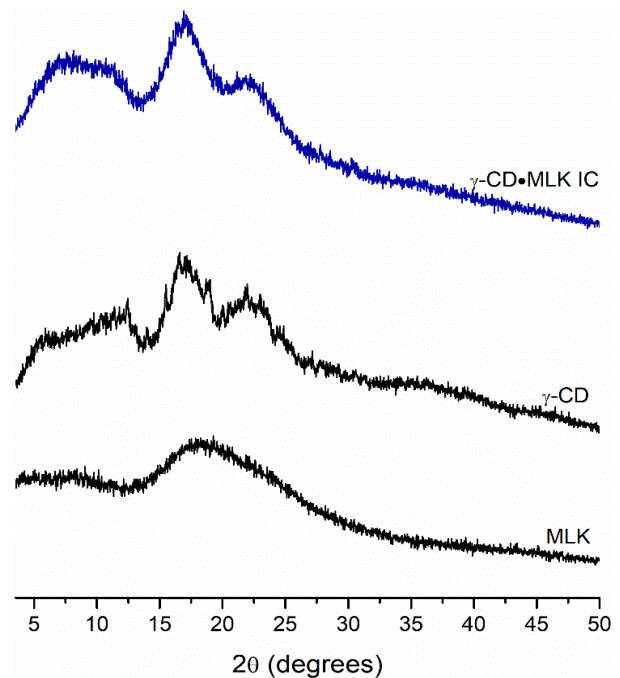

Figure 1. Powder X-ray diffraction patterns of (bottom) montelukast sodium (MLK), as received, (middle) amorphous $\gamma$-CD heptahydrate, and (top) $\gamma$-CD·MLK, prepared by mechanochemistry.
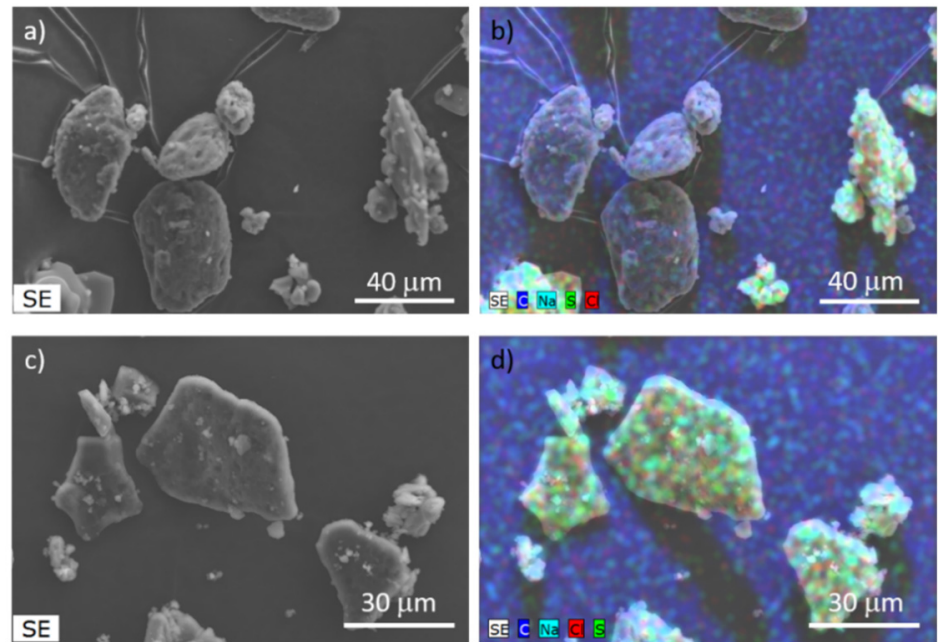

Figure 2. Scanning electron microscopy images and energy dispersive $X$-ray mapping of: a physical mixture of MLK and amorphous $\gamma$-CD ( $\mathbf{a}$ and $\mathbf{b}$, respectively) and of $\gamma$-CD.MLK prepared by mechanochemistry ( $\mathbf{c}$ and $\mathbf{d}$, respectively). EDS is shown to access the distribution of MLK characteristic elements: $\mathrm{S}, \mathrm{Na}$ and $\mathrm{Cl}$ atoms.

\subsection{Dissolution of $\gamma$-CD.MLK IC in Pure Water}

The dissolution profiles of both pure MLK and $\gamma$-CD·MLK were first studied in ultrapure water. In a previous study we had reported that till ca. $60 \mathrm{~min}$, the IC showed a slightly higher dissolution than pure MLK [22]. Similarly, herein we noticed that over $30 \mathrm{~min}$ more dissolved from $\gamma$-CD·MLK (Figure 3). 


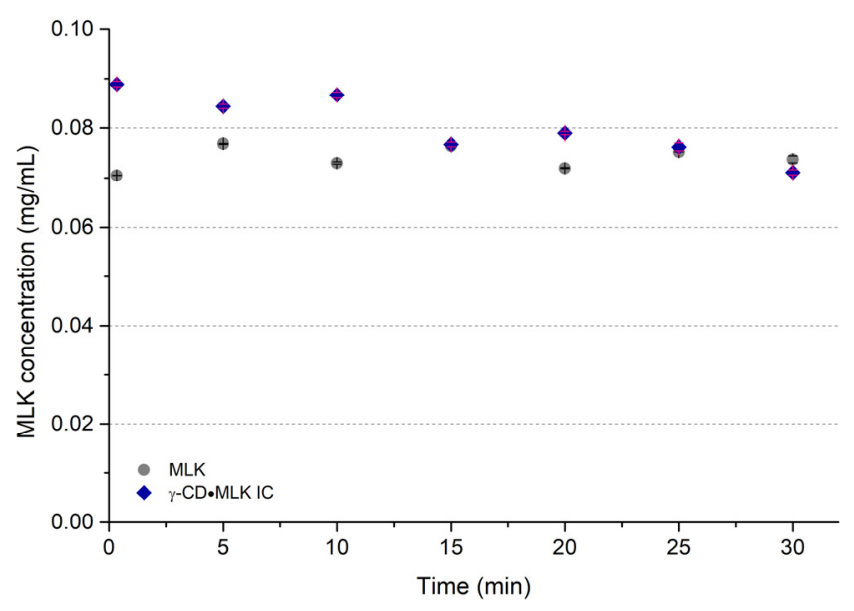

Figure 3. Preliminary investigation of MLK and $\gamma$-CD·MLK dissolution in ultrapure water.

Following these preliminary results, the dissolution profiles of pure MLK and $\gamma$-CD.MLK were evaluated under the guidelines of the US pharmacopea and the FDA for BCS class II drugs [27], i.e., in addition to the $30 \mathrm{~min}$ of analysis time, the apparatus, the volume of the medium, the drug dosage and the sampling times were adjusted as described in Section 2.5. Besides the recommended media (SDS $0.5 \%)$, pure water and aqueous buffers at $\mathrm{pH}$ values of 4.5 and 6.8 were also used.

In ultrapure water, MLK was practically unable to dissolve, both from pure MLK and from $\gamma$-CD.MLK. With pure MLK there was no trace of the drug dissolved in the medium; with $\gamma$-CD.MLK very low amounts of MLK were detected (Figure 4). In a way, these results are in accordance with those that we previously reported and showed that, at lower times, the dissolution profile of the pure drug versus the IC is similar, but the IC allows the dissolution of slightly higher amounts of MLK [22]. A completely different dissolution profile was seen with a solution of ultrapure water with $0.5 \%(\mathrm{~m} / \mathrm{v})$ of sodium dodecyl sulphate (SDS). In this medium, which is considered as a reference for dissolution studies of MLK, almost $100 \%$ of MLK dissolution was observed from both pure drug and $\gamma-$ CD·MLK. Besides, the dissolution profiles were very similar, almost equivalent, for both the pure drug and $\gamma$-CD.MLK (Figure 5).

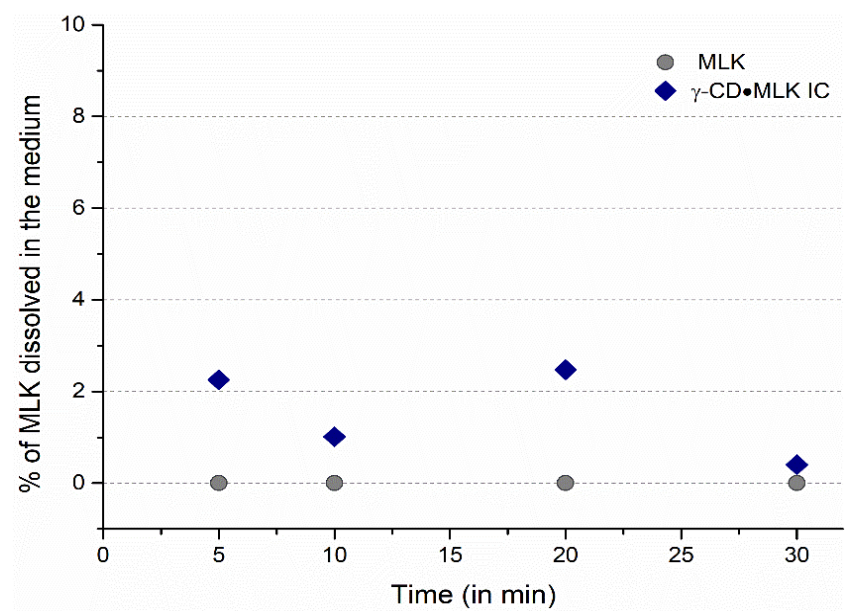

Figure 4. Dissolution profile of MLK and $\gamma$-CD·MLK in ultrapure water. 


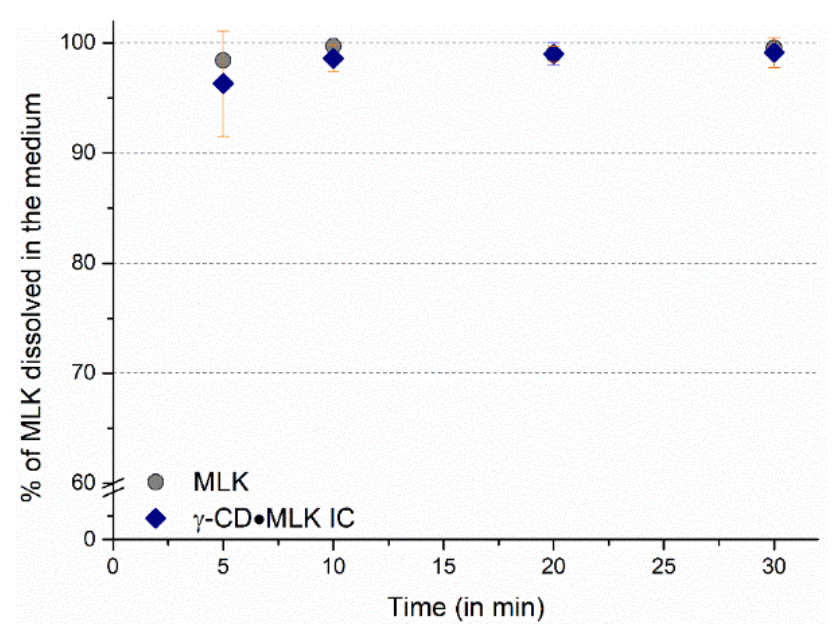

Figure 5. Dissolution profile of MLK and $\gamma$-CD.MLK in SDS $0.5 \%(\mathrm{~m} / \mathrm{v})$.

Additional studies were also made in media that simulate both the intestinal [28] and the duodenum environments. To mimic the intestinal environment, a solution of simulated intestinal fluid (without pancreatin), with $\mathrm{pH}$ buffered at 6.8 was used. In this medium the behavior of pure MLK and $\gamma$-CD.MLK was very similar to that previously observed in ultrapure water. While with pure MLK no drug was dissolved, with the IC a small amount of the drug was able to dissolve (Figure 6). In opposition to this, when in a medium of acetate buffer at $\mathrm{pH} 4.5$, which mimics the duodenum environment, neither pure MLK nor $\gamma$-CD·MLK evidenced any dissolution, as no traces of MLK were detected in the medium (Figure 7). This may be due to the low $\mathrm{pH}$ (4.5), as MLK is a weak and hydrophobic acid that does not dissolve in an acidic media.

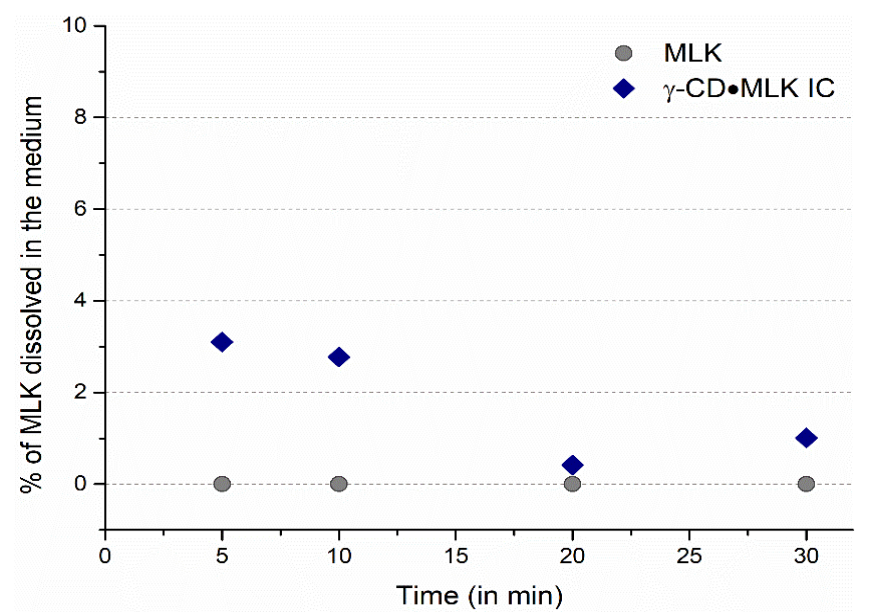

Figure 6. Dissolution profile of MLK and $\gamma$-CD.MLK in simulated intestinal fluid, that is, in a buffer at $\mathrm{pH} 6.8$. 


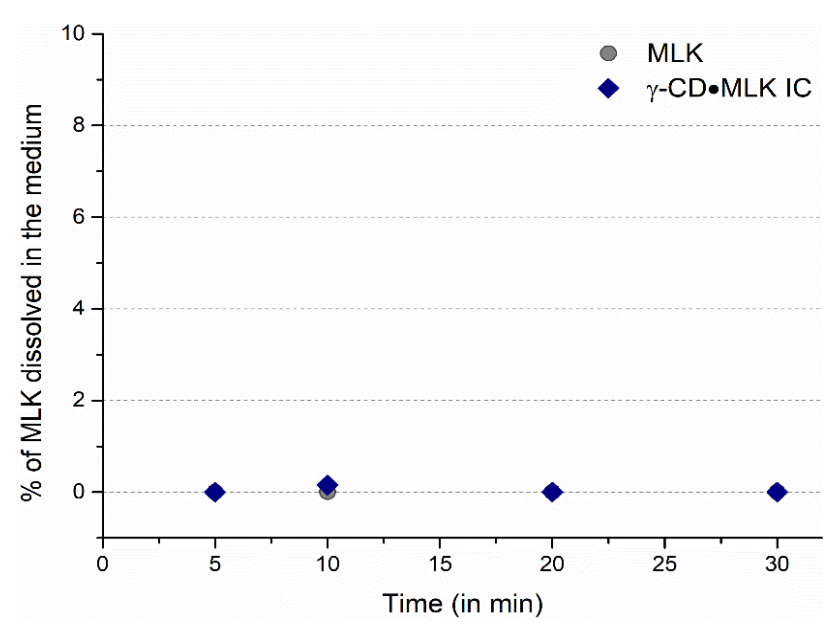

Figure 7. Dissolution profile of MLK and $\gamma$-CD.MLK in simulated duodenum environment (acetate buffer at $\mathrm{pH}$ 4.5).

\section{Conclusions}

This work confirmed the reproducibility of the preparation of $\gamma$-CD·MLK by mechanochemistry [22] by advanced characterisation techniques, such as PXRD and SEM.

A thorough analysis of the dissolution profile of both pure MLK and $\gamma$ CD·MLK was performed. When in ultrapure water or in a medium that simulates the intestinal environment, the IC tended to afford a slightly higher dissolution of MLK (ca. 3-5\%). Moreover, when in an SDS 0.5\%(m/v) solution, the pharmacopoeia reference medium for the MLK dissolution test, the dissolution profiles of pure MLK and $\gamma$-CD.MLK IC were practically identical, thus indicating bioequivalence. Similar profiles for pure MLK and $\gamma$-CD.MLK were also seen in the simulated duodenum environment, with both compounds showing no dissolution of the MLK.

Overall, this work provides insightful results on the use of mechanochemistry as a sustainable methodology to form inclusion compounds between $\gamma-C D$ and MLK. The prepared IC seems to be able to improve, at some extend, the dissolution of MLK in a few media, while having a dissolution profile equal to pure MLK in the reference medium and thus hinting at bioequivalence, a required trait for its incorporation into oral dosage forms. It is thus possible for $\gamma$-CD·MLK to help the pharmaceutical industry to overcome current setbacks and develop new MLK formulations with enhanced therapeutic effectiveness.

Data Availability Statement: All sata in this manuscript is proprietary of University of Aveiro and of JSB, FAAP and SSB.

Acknowledgments: Thanks are due to the University of Aveiro and Fundação para a Ciência e a Tecnologia for the financial support for the QOPNA research Unit (FCT UID/QUI/00062/2019), to the associated laboratory LAQV-REQUIMTE (project reference UIDB/50006/2020) and to CICECOAveiro Institute of Materials (UIDB/50011/2020 and UIDP/50011/2020), through national founds and, where applicable, cofinanced by the FEDER, within the PT2020 Partnership Agreement. We also thank FCT for the Ph.D. grant No. PD/BD/135104/2017 (to J.S.B.).

Conflicts of Interest: The authors declare no conflict of interest. 


\begin{abstract}
Abbreviations
API, active pharmaceutical ingredient; CD, cyclodextrin; COPD, chronic obstructive pulmonary disease; EDS, energy-dispersive X-ray spectroscopy; IC, inclusion compound; MLK, montelukast sodium; PXRD, powder X-ray diffraction; SDS, sodium dodecyl sulphate; SEM, scanning electron microscopy.
\end{abstract}

\title{
References
}

1. Singh, R.K.; Tandon, R.; Dastidar, S.G.; Ray, A. A review on leukotrienes and their receptors with reference to asthma. J. Asthma 2013, 50, 922-931.

2. Tenero, L.; Piazza, M.; Sandri, M.; Azzali, A.; Chinellato, I.; Peroni, D.; Boner, A.; Piacentini, G. Effect of montelukast on markers of airway remodeling in children with asthma. Allergy Asthma Proc. 2016, 37, 77-83.

3. Phipatanakul, W.; Nowak-Wegrzyn, A.; Eggleston, P.A.; Natta, M.V.; Kesavan, J.; Schuberth, K.; Wood, R.A. The efficacy of montelukast in the treatment of cat allergen induced asthma in children. J. Allergy Clin. Immun. 2002, 109, 794-799.

4. Price, D.; Popov, T.A.; Bjermer, L.; Lu, S.; Petrovic, R.; Vandormael, K.; Mehta, A.; Strus, J.D.; Polos, P.G.; Philip, G. Effect of montelukast for treatment of asthma in cigarette smokers. J. Allergy Clin. Immun. 2013, 131, 763-771.e766.

5. Hon, K.L.E.; Leung, T.F.; Leung, A.K.C. Clinical effectiveness and safety of montelukast in asthma. What are the conclusions from clinical trials and meta-analyses? Drug Des. Devel. Ther. 2014, 8, 839-850.

6. Morice, A.H.; Kastelik, J.A.; Aziz, I. Montelukast sodium in cystic fibrosis. Thorax 2001, 56, 244-244.

7. Lakomski, P.G.; Chitre, M. Evaluation of the utilization patterns of leukotriene modifiers in a large managed care health plan. J. Manag. Care Pharm. 2004, 10, 115-121.

8. De Lepeleire, I.; Reiss, T.F.; Rochette, F.; Botto, A.; Zhang, J.; Kundu, S.; Decramer, M. Montelukast causes prolonged, potent leukotriene D4-receptor antagonism in the airways of patients with asthma. Clin. Pharmacol. Ther. 1997, 61, 83-92.

9. Barbosa, J.S.; Almeida Paz, F.A.; Braga, S.S. Montelukast medicines of today and tomorrow: From molecular pharmaceutics to technological formulations. Drug Deliv. 2016, 23, 3257-3265.

10. Merck \& Co., Inc. Prescribing Information for Singulair (R). Available online: https://www.merck.com/research-andproducts/singulair/ (accessed on 12 November 2020).

11. Al Omari, M.M.; Zoubi, R.M.; Hasan, E.I.; Khader, T.Z.; Badwan, A.A. Effect of light and heat on the stability of montelukast in solution and in its solid state. J. Pharm. Biomed. Anal. 2007, 45, 465-471.

12. Kim, Y.H.; Kim, D.W.; Kwon, M.S.; Kwon, T.K.; Park, J.H.; Jin, S.G.; Kim, K.S.; Kim, Y.I.; Park, J.-H.; Kim, J.O.; Yong, C.S.; Woo, J.S.; Choi, H.-G. Novel montelukast sodium-loaded clear oral solution prepared with hydroxypropyl- $\beta$-cyclodextrin as a solubilizer and stabilizer: Enhanced stability and bioequivalence to commercial granules in rats. J. Incl. Phenom. Macrocycl. Chem. 2015, 82, 479-487.

13. Rashmitha, N.; Raj, T.J.S.; Srinivas, C.; Srinivas, N.; Ray, U.K.; Sharma, H.K.; Mukkanti, K. A Validated RP-HPLC Method for theDetermination of Impurities in Montelukast Sodium. E-J. Chem. 2010, 7, 156593.

14. Kwon, T.K.; Choi, Y.K.; Yan, X.W.; Wang, Z.Z.; Kim, Y.I.; Park, J.H.; Woo, J.S. Liquid Formulation Comprising Montelukast or Pharmaceutically Acceptable Salt Thereof and Method for Preparing Same. Patent WO 2015093847-A1, 25 June 2015.

15. Uekama, K.; Hirayama, F.; Irie, T. Cyclodextrin Drug Carrier Systems. Chem. Rev. 1998, 98, $2045-2076$.

16. Liu, L.; Zhu, S. Preparation and characterization of inclusion complexes of prazosin hydrochloride with $\beta$-cyclodextrin and hydroxypropyl- $\beta$-cyclodextrin. J. Pharm. Biomed. Anal. 2006, 40, 122-127.

17. Evrard, B.; Chiap, P.; DeTullio, P.; Ghalmi, F.; Piel, G.; Van Hees, T.; Crommen, J.; Losson, B.; Delattre, L. Oral bioavailability in sheep of albendazole from a suspension and from a solution containing hydroxypropyl-beta-cyclodextrin. J. Control. Release 2002, 85, 45-50.

18. Wong, J.W.; Yuen, K.H. Improved oral bioavailability of artemisinin through inclusion complexation with $\beta$ - and $\gamma$ cyclodextrins. Int. J. Pharm. 2001, 227, 177-185.

19. Shah, N.; Seth, D.; Balaraman, R. Bioavailability enhancement of poorly soluble raloxifene by designing inclusion complex with $\beta$-cyclodextrin. Int. J. Pharm. Pharm. Sci. 2015, 7, 205-211.

20. Im, H.T.; Rim, H.P.; Kim, Y.I.; Park, J.H.; Woo, J.S. Complex Granule Formulation Having Improved Stability Comprising Levocetirizine and Montelukast. Patent WO 2014208915-A2, 31 December 2014.

21. Durán Merás, I.; Espinosa-Mansilla, A.; Rodríguez, D.A. Complexation study of cinalukast and montelukast with cyclodextrines. J. Pharm. Biomed. Anal. 2007, 43, 1025-1032.

22. Barbosa, J.S.; Nolasco, M.M.; Ribeiro-Claro, P.; Almeida Paz, F.A.; Braga, S.S. Preformulation Studies of the y-Cyclodextrin and Montelukast Inclusion Compound Prepared by Comilling. J. Pharm. Sci. 2019, 108, 1837-1847.

23. Dushkin, A. Potential of mechanochemical technology in organic synthesis and synthesis of new materials. Chem. Sustain. Dev. 2004, 12, 251-273.

24. Wang, Q.; Li, S.; Che, X.; Fan, X.; Li, C. Dissolution improvement and stabilization of Ibuprofen by co-grinding in a $\beta$ cyclodextrine ground complex. Asian J. Pharm. Sci. 2010, 5, 185-190.

25. Aigner, Z.; Berkesi, O.; Farkas, G.; Szabó-Révész, P. DSC, X-ray and FTIR studies of a gemfibrozil/dimethyl- $\beta$-cyclodextrin inclusion complex produced by co-grinding. J. Pharm. Biomed. Anal. 2012, 57, 62-67. 
26. Inoue, Y.; Watanabe, S.; Suzuki, R.; Murata, I.; Kanamoto, I. Evaluation of actarit $/ \gamma$-cyclodextrin complex prepared by different methods. J. Incl. Phenom. Macrocycl. Chem. 2015, 81, 161-168.

27. US Food and Drug Administration. Dissolution Methods. US Department of Health and Human Services, FDA. Available online: https://www.accessdata.fda.gov/scripts/cder/dissolution/dsp_getalldata.cfm (accessed on 24 April 2019).

28. US Food and Drug Administration. Guidance for Industry: Bioavailability and Bioequivalence Studies for Orally Administered Drug Products-General Considerations; US Department of Health and Human Services, FDA, Center for Drug Evaluation and Research: Rockville, MD, USA, 2003. 